\section{Trabeculectomy: a training dilemma}

\author{
R Troutbeck ${ }^{1}$, GA Lee ${ }^{1,2}$, P Sanfilippo ${ }^{3}$ and \\ B Fleming ${ }^{1}$
}

\begin{abstract}
Aim/Purpose The incidence of trabeculectomy is falling due to more effective medical therapies, resulting in fewer trabeculectomy operations carried out by Ophthalmology trainees. This study audits all primary trabeculectomy procedures performed publicly at the two major teaching hospitals in Brisbane, Australia.

Method A retrospective consecutive case review analysing the rates and outcomes of Ophthalmology trainee versus consultant trabeculectomies that were performed during the period May 1996-May 2002.

Results In all, 290 primary trabeculectomies were undertaken during the study period. Ophthalmology trainees performed 95 (33\%) of the procedures. This represents a rate of $\mathbf{1 . 6}$ trabeculectomy procedures per year per trainee (10 trainees train at the two hospitals per year). Rates of complications postoperatively were similar between the trainee and consultant groups. The percentage of patients achieving an IOP $\leqslant 15 \mathrm{mmHg}$ on no medication at their last follow-up was low at $30 \%$.

Conclusions The results of this study suggests that improvement in trabeculectomy technique is required. In order to acquire skills, wetlab training on artificial or animal eyes may become mandatory within the training programmes, otherwise trabeculectomy will become the realm of subspecialists with glaucoma fellowship training. Eye (2007) 21, 183-186. doi:10.1038/sj.eye.6702159; published online 2 December 2005
\end{abstract}

Keywords: trabeculectomy; training programme; glaucoma treatment; wetlab

\section{Introduction}

The worldwide literature suggests that the incidence of trabeculectomy is decreasing. This has been contributed by the advent of more effective medical therapies. ${ }^{1-4}$ The Collaborative
Initial Glaucoma Treatment study has found that although surgery lowers intraocular pressure (IOP) more than medical therapy, no significant difference is found in visual field progression over the course of 5 years. ${ }^{5}$ Despite these trends, trabeculectomy remains a critical treatment modality in glaucoma for those patients on maximum medical therapy with field progression, uncontrolled pressure and/or progressive nerve damage. In these patients, drainage surgery must achieve a stable reduction in IOP. The limited number of trabeculectomies being performed however, results in reduced surgical competency and poorer experience in the equally important postoperative period. Not only are trained ophthalmologists losing this expertise, but the trainees are not acquiring the necessary skills.

\section{Methods}

A retrospective study of all adult trabeculectomies performed publicly from May 1996 to April 2002 at the two main teaching hospitals in Brisbane, Queensland - Royal Brisbane Hospital and the Princess Alexandra Hospital was conducted. The rates and outcomes of trainee performed primary trabeculectomies were compared with the consultant group (consisting of experienced fellowship level ophthalmologists and including one subspecialty trained consultant in glaucoma). Trainee cases were defined as being performed by the trainee as sole operator with or without supervision, while consultant cases were defined as being performed by the consultant in total or by the trainee in part under close supervision. Outcomes and rates of complications were compared between trainees and consultants. Probability values $<0.05$ were regarded as statistically significant.

\section{Results}

The total number of trabeculectomies performed publicly from May 1996 to May 2002 was 352. Of these, 290 (82\%) were primary
${ }^{1}$ Royal Brisbane Hospital, Brisbane, Australia

${ }^{2}$ Department of Ophthalmology, University of Queensland, Brisbane, Australia

${ }^{3}$ Brisbane North Eye Centre, Brisbane, Australia

Correspondence: G Lee, City Eye Centre, 10/135 Wickham Tce, Brisbane, Qld 4000, Australia

Tel: + 61738316888 ;

Fax: +61 738316883 ;

E-mail: eye@

cityeye.com.au

Received: 23 June 2005 Accepted in revised form: 17 September 2005 Published online: 2 December 2005

There were no competing interests by the authors in the production of this manuscript 


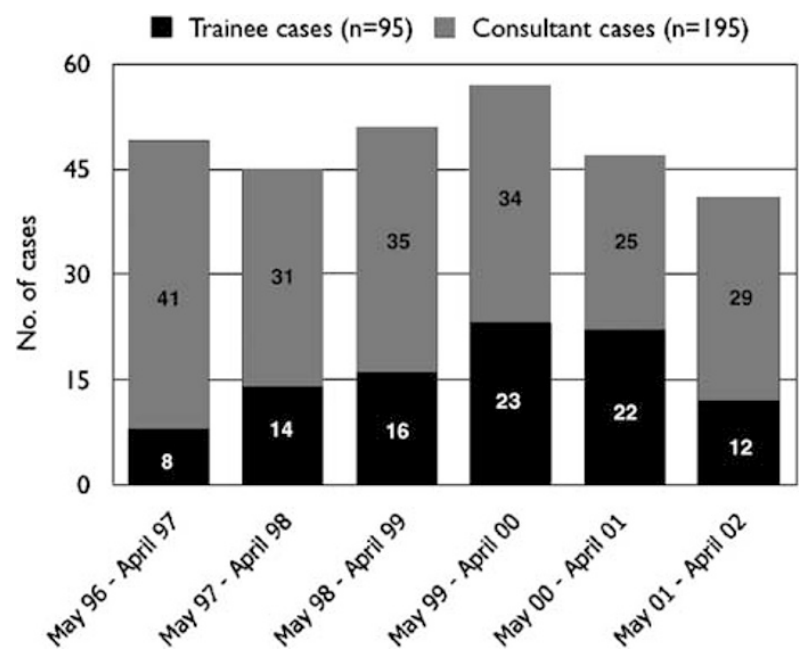

Figure 1 Rate of trabeculectomy.

procedures, with $95(33 \%)$ performed by the trainees. In all, 151 (52\%) patients were male, 276 (95\%) Caucasian and the average age was 69 years (range 19-93 years). The average length of follow-up was 20 months (range 6 months to 7 years). All second or subsequent operations were performed by the consultants.

The rate of trabeculectomy being performed by trainees ranged from 8 to 23 per year over the surveyed time period (Figure 1). This data shows a fluctuating number of primary procedures being performed per year, with the actual number of trainee procedures averaging only 1.6 procedures per year (taking into account that 10 trainees train at each of the two teaching hospitals per year). Trabeculectomy with 5-fluorouracil (5-FU) (98; 36 trainee : 62 consultant) was the most commonly performed surgery, followed by phacotrabeculectomy (90; 27 trainee: 63 consultant), trabeculectomy without antimetabolite (53; 22 trainee: 31 consultant) and other combined trabeculectomy procedures (28). Trabeculectomy with mitomycin-C (21; 21 trainee: 20 consultant) was performed much less commonly and these cases were almost exclusively performed by consultants, which is indicative of greater complexity of these cases.

The most common complications encountered during the first postoperative week were wound leak (55), flat anterior chamber (25), IOP $<5 \mathrm{mmHg}$ (56), IOP $>25 \mathrm{mmHg}$ (89) and hyphaema (45) (Figure 2).

Complication rates in this period showed no significant difference between trainees and consultants with at least one of the above complications occurring in $62(65 \%)$ of the 95 trainee cases compared with $121(62 \%)$ of the 195 consultant cases $(P=0.30)$.

The outcomes of success for trabeculectomy that can be considered include IOP lowering, minimization of visual field progression and optic nerve stabilization.

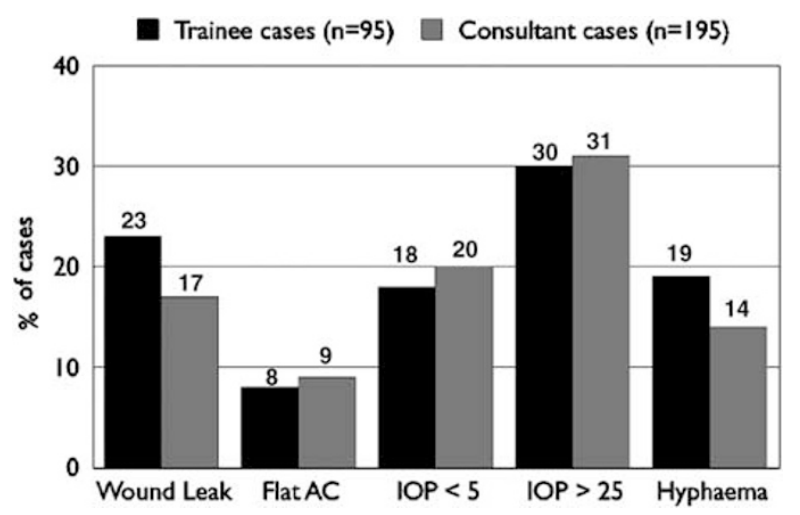

Figure 2 Complications in the first postoperative week.

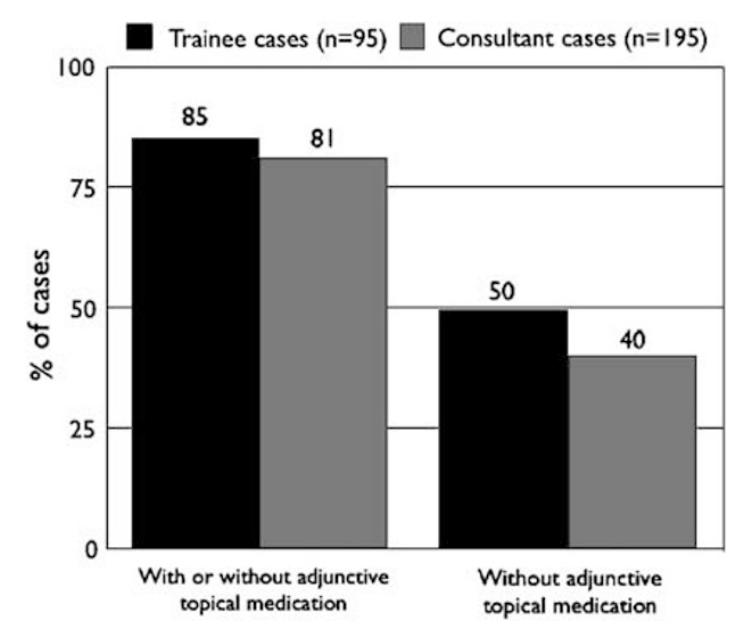

Figure 3 Cases achieving IOP $\leq 21 \mathrm{mmHg}$.

Unfortunately, in this cohort of patients pre and postoperative visual fields was performed in only 99 (34\%) of patients. No serial optic disc photography was performed as found included in the patient files. The IOP at last follow-up for primary trabeculectomy was compared between trainee and consultant cases (Figures 3 and 4$)$. An IOP of $\leq 21$ was achieved in $239(82 \%)$ of cases, however, only $125(43 \%)$ reached this target on no postoperative medication. A target pressure of $\leq 15 \mathrm{mmHg}$, a level regarded as necessary for stabilisation of the visual field in advanced glaucoma was achieved in $148(51 \%)$ patients with or without medication (Figure 4) Only $88(30 \%)$ of patients reached this level of IOP on no medication. There were no significant differences between the IOP achieved by the trainees compared with the consultants at IOP $\leq 21$ $(P=0.42)$ and at $\mathrm{IOP} \leq 15(P=0.29)$.

\section{Discussion}

Trabeculectomy surgery is technically demanding, requiring careful tissue handling and suturing 


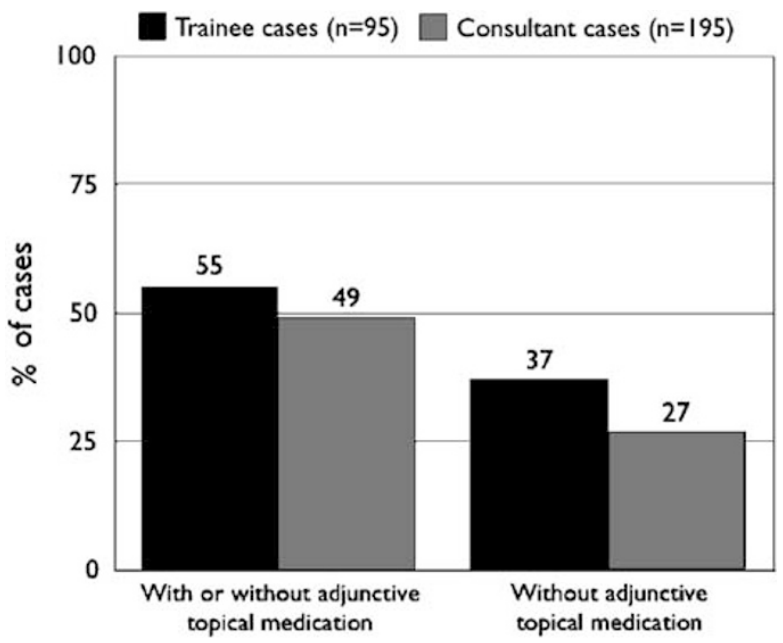

Figure 4 Cases achieving IOP $\leq 15 \mathrm{mmHg}$.

proficiency. In comparison, the majority of the surgery performed by trainees in teaching hospitals is clear corneal phacoemulsification requiring none or one corneal suture. Consequently, trainees are receiving limited exposure to the necessary skills. A major challenge is that by the time the decision is made to proceed to trabeculectomy, often the glaucoma is advanced, not responsive to medical therapy and hence long-term IOP control is defined by the surgical outcome. The first procedure is often the best chance to establish a functioning bleb as further surgery is often complicated by scar tissue. To establish a functioning bleb

comprehensive surgical experience is mandatory. In this study, relatively few patients underwent trabeculectomy with mitomycin-C compared to the number of 5-FU augmented cases. This is a reflection of the more conservative approach by this cohort of general-trained ophthalmologists. As the number of fellowship-trained surgeons in glaucoma increases then it is likely that greater potency antimetabolites will be utilised more frequently.

Our data showed similar results between consultant and trainee groups in terms of the first week complications and the outcomes achieved. This indirectly suggests that trainees are performing appropriate case complexity and are receiving adequate consultant supervision during the operation and in the subsequent postoperative period. It also indicates the consultants are performing the more complex cases, with the potential for more postoperative complications and less satisfactory outcomes. The rate of complications for the trabeculectomy procedures is high in the first postoperative week. Other studies have looked at early and late complications of trabeculectomy. ${ }^{6}$ Edmunds et al found $46.6 \%$ of cases had early complications - the most common of which were hyphaema, shallow AC and wound leak. Such a high rate of early complications suggests surgical techniques can be improved and the need for intensive follow-up of patients in this early postoperative period. The standard for current trabeculectomy procedure using limbal based flaps, interrupted scleral trapdoor sutures and single pledget sponges for antimetabolite application, lags behind other procedures in ophthalmology such as phacoemulsification which in comparison is associated with far fewer complications and less intensive follow-up.

In terms of monitoring glaucoma progression, there is a distinct paucity of visual field information in this cohort of patients treated within the public system. This is perhaps a reflection of the limited resources and time available in the public hospital system, whereas visual fields assessment and other additional tests (disc photography, nerve fibre layer thickness analysis) are more likely to be performed in private practice. Reassessment and objective follow-up investigations are mandatory in the management of glaucoma and should not be compromised whether in public or private healthcare.

The results of $30 \%$ of patients obtaining a final target IOP of $\leq 15 \mathrm{mmHg}$ are relatively poor, if this level is considered as success on no medication. These results are considerably worse than those reported elsewhere in the literature. ${ }^{6,7}$ However, it must be noted that our audit included all trabeculectomy cases undertaken on a variety of different glaucoma types, using different techniques and a variety of ethnic groups. Obviously, improvement in surgical technique is required as well as more diligent postoperative follow-up.

An important issue to consider is the decreased numbers of procedures likely to be performed in the future. Walland et $a l^{3}$ looked at glaucoma treatment in Australia. He found a significant decrease in the rate of trabeculectomy in Australia over the period between 1994 and 2003. This suggests that the issue of providing adequate training for trainees is a national problem. The international experience appears similar with Franks finding a decline in trabeculectomy rates at Moorfields Eye Hospital, London, UK from 1997 to 2002.

In Brisbane, a permanent wetlab has been set-up for trainees to practice trabeculectomy procedures on pigs' eyes. In order to acquire skills, wetlab training on artificial or animal eyes may become a mandatory part of the curriculum. Lee et $a l^{8}$ have developed a teaching model using pig eyes prepared in formalin which enables trainee surgeons to practice the various aspects of tissue handling required for successful surgery. Unless adequate experience is obtained during their general ophthalmic training, then trabeculectomy will become 
the realm of subspecialists with glaucoma fellowship training. Clearly as glaucoma is a common disease in an ageing population, there will be a significant shortage of surgical manpower unless training standards can be improved.

\section{References}

1 Whittaker KW, Grillow JT, Cunliffe IA. Is the role of trabeculectomy in glaucoma management changing? Eye 2001; 15: 449-452.

2 Bateman DN, Clark R, Azuara-Blanco A, Bain M, Forrest J. The effects of new topical treatments on the management of glaucoma in Scotland: an examination of ophthalmological health care. Br J Ophthalmol 2002; 86: 551-554.
3 Walland MJ. Glaucoma treatment in Australia: changing patterns of therapy 1994-2003. Clin Experiment Ophthalmol 2004; 32: 590-597.

4 Franks W. Glaucoma surgery at Moorfields: an analysis of the changing patterns of glaucoma surgery from 1997 to 2003. ANZ Glaucoma Club Proceedings, Melbourne; 2004; 2-5.

5 Lichter PR, Musch DC, Gillespie BW et al. Interim clinical outcomes in the collaborative initial glaucoma treatment study comparing initial treatment randomized to medication or surgery. Ophthalmology 2001; 108: 1943-1953.

6 Edmunds B, Thompson JR, Salmon JF et al. The national survey of trabeculectomy 111. Early and late complications. Eye 2002; 16: 297-303.

7 Anand N, Mielke C, Dawda VK. Trabulectomy outcomes in advanced glaucoma in Nigeria. Eye 2001; 15: 274-278.

8 Lee GA, Chiang MY, Shah P. Pig eye trabeculectomy-a wetlab teaching model. Eye. 2005 Jan 28 (E-pub ahead of print). 